\title{
High Throughput Intact Protein Analysis Using Infrared Matrix-Assisted Laser Desorption Electrospray Ionization Mass Spectrometry
}

Fan $\mathrm{Pu}^{1}$, Scott A. Ugrin ${ }^{1}$, Andrew J. Radosevich ${ }^{1}$, David Chang-Yen ${ }^{1}$, James W. Sawicki ${ }^{1}$, Nari N. Talaty ${ }^{1}$, Nathaniel L. Elsen ${ }^{1} *$ and Jon D. Williams ${ }^{1} *$

1. AbbVie Inc., 1 North Waukegan Rd., North Chicago, IL 60064

Corresponding authors:

Nathaniel L. Elsen

Email: Nathaniel.elsen@abbvie.com

Jon D. Williams

Email: Jon.williams@abbvie.com

\section{Graphical Abstract}

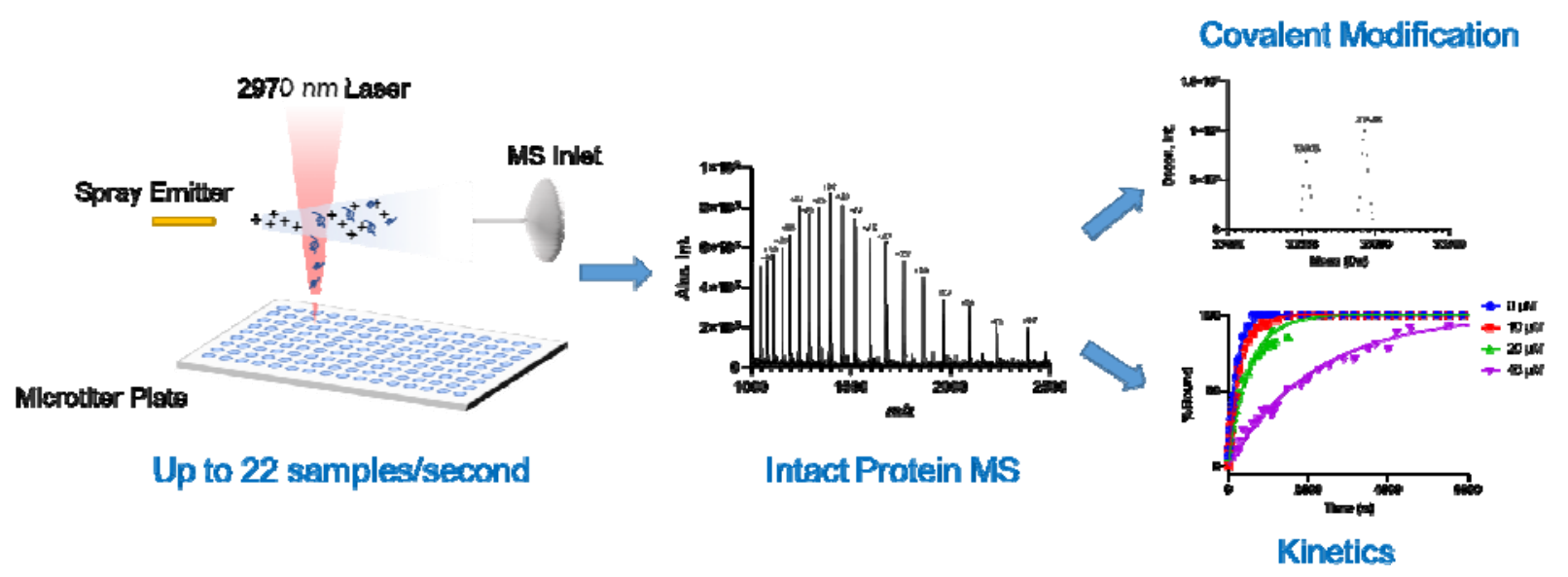




\begin{abstract}
:
Mass spectrometry (MS) is the primary analytical tool used to characterize proteins within the biopharmaceutical industry. Electrospray ionization (ESI) coupled to liquid chromatography is the gold standard technique for intact protein analysis. However, speed limitations prevent analysis of large sample numbers (>1000) in a day. Infrared matrix-assisted laser desorption electrospray ionization (IRMALDESI) MS, an ambient ionization MS technology, has recently been established as a platform for high throughput small molecule analysis. Here we report the application of such system for intact protein analysis. Up to $150 \mathrm{kDa}$ proteins are detectable on a high throughput IR-MALDESI-MS system and we have evaluated how matrix affects signal. The system can analyze up to 22 protein samples in a second. Protein autophosphorylation, compound binding kinetics and compound modifications to a probe protein are demonstrated as applications. Top-down protein sequencing was conducted to identify a cysteine modification site. Two plate-based high throughput sample cleanup methods were coupled to IRMALDESI-MS to enable analysis of samples containing high amounts of salts and buffers without compromising speed.
\end{abstract}

\title{
Introduction:
}

A wide variety of recombinant proteins are produced for drug discovery in the biopharmaceutical industry and require identity confirmation and characterization. Target proteins are developed as reagents for small molecule drug discovery to perform screening assays to find hit and lead compounds and to conduct structural biology studies for structure guided design. The amount and extent of target protein modification by phosphorylation, or other post-translational modifications, or covalent small molecule inhibitors is determined to ensure protein is in an active form or to enable finding new covalent hit compounds. Therapeutic proteins such as monoclonal antibodies, dual variable domain antibodies, bispecific antibodies, and fusion proteins typically have glycosylated forms. The type and amount of glycosylation are determined along with confirmation or protein identity. Many of these therapeutic proteins can then be conjugated with drug and a linker to form an antibody drug conjugate (ADC). The extent of conjugate drug-to-antibody ratio (DAR) is typically measured by mass spectrometry (MS).

MS has become the primary analytical tool used to characterize both target and therapeutic proteins since the invention of electrospray ionization (ESI) ${ }^{1}$ and matrix assisted laser desorption ionization (MALDI) ${ }^{2}$ sources, which still remain the primary methods for ionizing proteins. ${ }^{3}$ MALDI can analyze samples rapidly at a rate of $<1$ s/sample when coupled to a time-of-flight (TOF) mass spectrometer and produces primarily singly charged ions, which enables direct determination of the molecular weight. However, proteins are desalted, and crystallized with matrix before introduction to the instrument, which prohibits further measurement of kinetics, and the mass resolution is insufficient to assign accurate mass assignments on large proteins with small mass modifications. In contrast, ESI produces multiply charged ions, which requires a deconvolution algorithm to transform the signal from the $\mathrm{m} / \mathrm{z}$ to the mass domain. Despite this issue, proteins produced by ESI can be coupled to higher resolution mass spectrometers such as orthogonal acceleration TOF (OA-TOF), orbitrap (OT), or Fourier Transform - Ion Cyclotron Resonance (FT-ICR) instruments to produce sufficient resolution to detect small mass changes on large proteins.

ESI sources are typically coupled to reversed-phase liquid chromatography (LC) to enable online desalting and if necessary, to separate proteins. For simple recombinant proteins, LC-MS runs of 1 5 minutes can be conducted routinely using $<10$ pmol of material injected onto the column and be set-up as a part of a fully integrated protein open-access LC-MS system. ${ }^{4}$ These systems are robust and are 
useful for the analysis of up to 384 samples. However, for some activities, where $1000-10,000$ samples require analysis, faster and more comprehensive MS analysis techniques are needed.

Progress toward high-throughput protein MS analysis was demonstrated by Campuzano et al. using a RapidFire HT-MS system coupled to an OA-TOF system to achieve a sampling rate of 20 s/sample ${ }^{5}$. They utilized this system to screen the reactivity of 1000 acrylamide compounds after incubation with a $19.5 \mathrm{kDa}$ protein. Sawyer et al., developed a high throughput antibody screening platform with a RapidFire system coupled to an OA-TOF which had a 25 s cycle time between injections and was capable of running 3400 samples per day. ${ }^{6}$ They also showed that coupling of the RapidFire to an Orbitrap Exploris 480 could be done and led to better resolution on glycoforms. Another sample introduction device, the SampleStream, has been developed, which can deliver denatured or native state proteins to a MS system at $15 \mathrm{~s} / \mathrm{sample}^{7}$

Faster sample rates $(<1 \mathrm{~s} / \mathrm{sample})$ have been demonstrated for the analysis of small molecules and have been adopted for biochemical and cell-based high-throughput screening assays using various techniques including acoustic droplet mist $^{8}$, acoustic droplet - open port injection ${ }^{9}$, desorption electrospray ionization (DESI) ${ }^{10}$ and infrared matrix assisted laser desorption electrospray ionization (IRMALDESI $)^{11}$. These techniques do not require a separation step prior to introduction to the mass spectrometer. Each technique experiences a reduction in signal compared to LC/MS, but for most screening assays the signal-to-noise is sufficient to generate robust data.

Multiple studies have been conducted demonstrating the ability of ambient ionization techniques - DESI, nanoDESI, and IR-MALDESI - to ionize proteins. DESI has been demonstrated for measurement of denatured proteins from $12 \mathrm{kDa}$ to $150 \mathrm{kDa}^{12-14}$ and an experiment was conducted to analyze proteins directly from tissue sections. ${ }^{15}$ Native MS with DESI has also been reported recently. ${ }^{16,17}$ With nanoDESI, MS imaging of proteins in tissue has also been demonstrated. ${ }^{18,}{ }^{19}$ Cooper et al. reported the first native-MS imaging of proteins using nanoDESI. ${ }^{20}$ Early work by Muddiman et al. showed that MALDESI is capable of intact and top-down protein analysis ${ }^{21,22}$ and Nemes et al., using LAESI (laser ablation electrospray ionization), were able to analyze bovine serum albumin (BSA) ${ }^{23}$.

Motivated by a need for higher throughput protein assays, the recent construction of a highthroughput IR-MALDESI-MS platform ${ }^{11}$, and the potential of analyzing proteins with ambient ionization, we set out to test capabilities of our platform for protein analysis. The performance of the system was established, including how matrix components affect the analysis. With improvements in syncing laser firing to triggering of the MS system, it is now feasible to analyze a 384 well plate of proteins in 17 seconds $(22.7 \mathrm{~Hz})$ with $<1 \%$ cross-contamination between wells. We will demonstrate various applications (autophosphorylation, compound binding kinetics, and covalent compound additions) that take advantage of the rapid analysis speed and show how this instrumentation can also be used to conduct top-down protein sequencing to facilitate identification of site modifications. Finally, we will demonstrate how simple and rapid plate-based desalting methods ( $<10$ minutes) can be added to improve sensitivity for samples in complex matrices.

\section{Results and Discussion:}

\section{Detectable Molecular Weight Range and Matrix Effect}

To establish the detectable molecular weight range of proteins with IR-MALDESI, we first analyzed protein standards ranging from $12 \mathrm{kDa}$ to $150 \mathrm{kDa}$ (cytochrome $\mathrm{C}$, carbonic anhydrase, recombinant human serum albumin/rHSA and NISTmAb). The protein standards were either dissolved in water (cytochrome $\mathrm{C}$ and carbonic anhydrase), diluted in water from buffered solution (rHSA) or buffer 
exchanged to $100 \mathrm{mM}$ ammonium acetate (NISTmAb). Raw mass-to-charge spectra are shown in Figure 1 with deconvoluted mass spectra as insets. Multiple glycoforms could be observed in the deconvoluted spectrum of NISTmAb (Figure S1). The spectra were collected using a resolving power of 7, 500 (FWHM at $\mathrm{m} / z=200$ ) and provided sufficient information for most studies. The detection limits of neat protein samples in water were $45 \mathrm{nM}$ and $136 \mathrm{nM}$ for cytochrome $\mathrm{C}$ and carbonic anhydrase, respectively. Higher resolving power can be used to resolve isotopic peaks (Figure S2a and b) when necessary, but results in a decreased deconvoluted protein intensity, as exemplified by a study using NISTmAb (Figure S2c-e).

By reducing NISTmAb with 5mM TCEP, we were able to detect light chains and heavy chains simultaneously. Measurements were taken repetitively and started as soon as possible after TCEP was added to NISTmAb. A shift in heavy chain/light chain ratio was observed. Light chain was detected at the first time point while heavy chain was still missing (Figure 2a). Peaks corresponding to two heavy chains linked together could be found at early time points which then converted to single heavy chain peaks (Figure 2a-c). The transition was completed in about 10 minutes and the heavy chain/light chain ratio remained stable afterwards (Figure 2d). Glycoforms of heavy chain could be resolved after deconvolution (Figure S3).

Previously it has been established that IR-MALDESI is compatible with complex matrices for small molecule analysis. ${ }^{11,}{ }^{24}$ In this study, we tested signal suppression effects from several common buffer components for protein analysis with cytochrome $\mathrm{C}$ and carbonic anhydrase (both at $11 \mu \mathrm{M}$ ). Three-fold serial titrations of matrices were performed individually with $\mathrm{NaCl}, \mathrm{MgCl}_{2}$, Tris $(\mathrm{pH}=7.5)$ and HEPES $(\mathrm{pH}=8)$ starting at $900 \mathrm{mM}$, Tween-20 at $0.9 \%$ and glycerol at $9 \%$. Maximum titrated concentrations of matrices at which could still produce deconvolute protein mass spectra were defined as tolerable limits. 
(a)

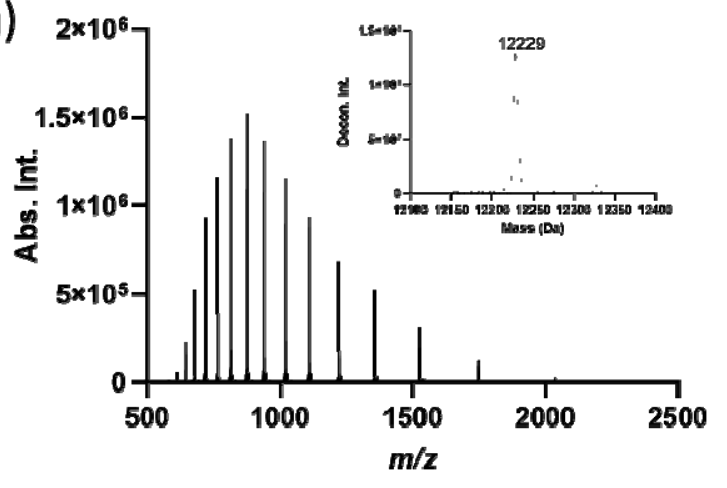

(c)

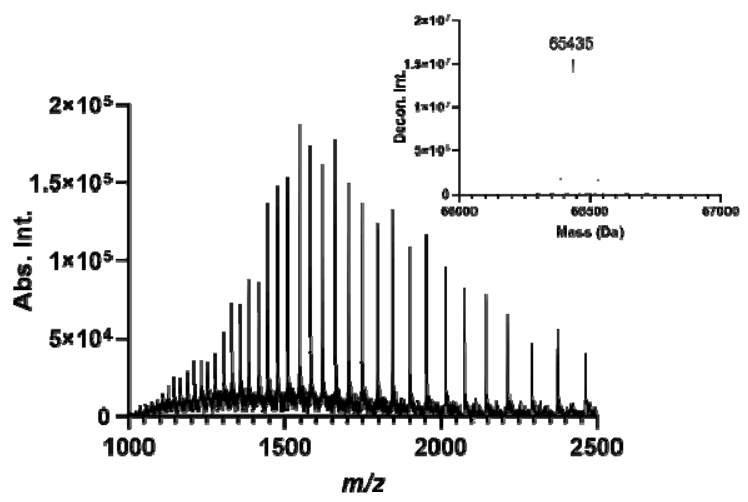

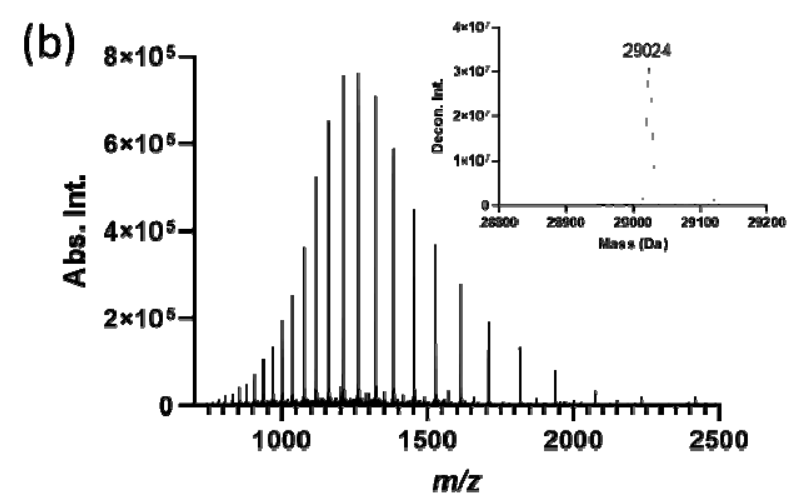

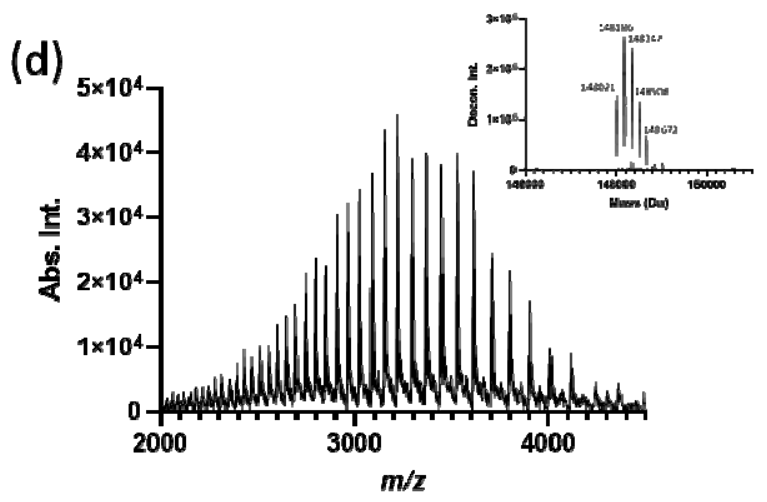

Figure 1. Protein mass spectra of standards. (a) $0.2 \mathrm{mg} / \mathrm{mL}$ cytochrome C; (b) $5 \mathrm{mg} / \mathrm{mL}$ carbonic anhydrase; (c) $3 \mathrm{mg} / \mathrm{mL}$ recombinant human serum albumin (diluted in water from buffered stock solution); (d) $10 \mathrm{mg} / \mathrm{mL}$ NISTmAb (buffer exchanged to 100 mM ammonium acetate). Insets: deconvoluted spectra.

The results are summarized in Table 1 . We observed concentration-response effects for $\mathrm{NaCl}, \mathrm{MgCl}_{2}$, Tris and HEPES (Figure S4a, b). These salts and buffers were well tolerated at mM levels. Tween-20 was not well tolerated, which indicates it should be avoided or removed during sample preparation. Glycerol had no significant ion suppression effect within the tested range (Figure S4c, d). 


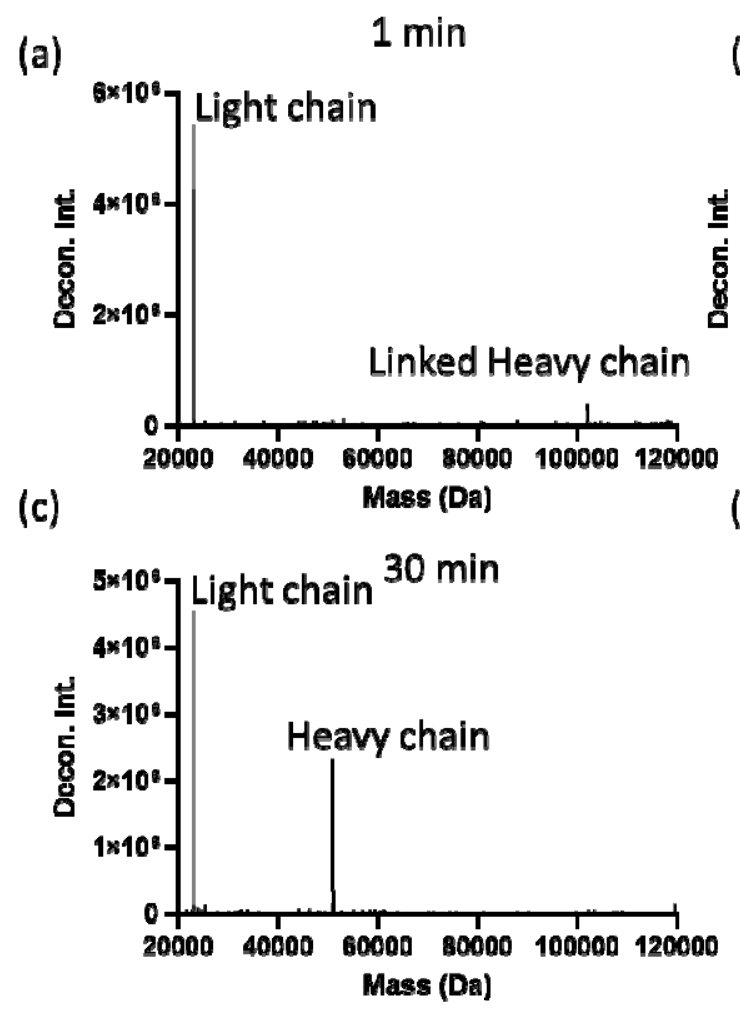

(b) $\quad 4 \mathrm{~min}$
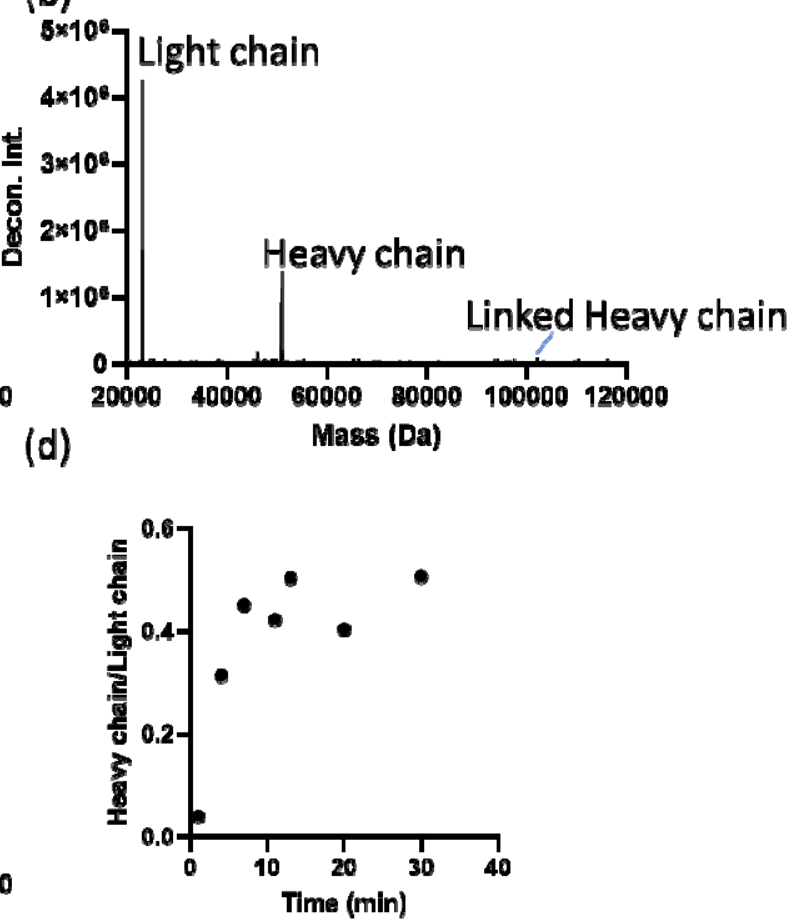

Figure 2. (a)-(c) Deconvoluted spectra at $1 \mathrm{~min}, 4 \mathrm{~min}$ and $30 \mathrm{~min}$ after adding TCEP to NISTmAb; (d) Heavy chain/light chain ratio after adding TCEP.

Table 1. IR-MALDESI-MS matrices tolerance for Cytochrome C and Carbonic Anhydrase.

Tolerable Limits Cytochrome C Carbonic Anhydrase

\begin{tabular}{lll}
\hline $\mathrm{NaCl}(\mathrm{mM})$ & 300 & 100 \\
\hline $\mathrm{MgCl}_{2}(\mathrm{mM})$ & 100 & 100 \\
\hline Tris $(\mathrm{pH} 7.5)(\mathrm{mM})$ & 300 & 300 \\
\hline HEPES (pH 8) (mM) & 100 & 33 \\
\hline Tween-20 (\%) & $0.01 \%$ & N/A \\
\hline Glycerol (\%) & $9 \%$ & $9 \%$ \\
\hline
\end{tabular}

\section{High Throughput Protein Analysis}

Having established IR-MALDESI-MS for measurement of protein standards, we investigated its capability for high throughput protein analysis. The buffer tolerance tests (see above) were performed using 384-well plates with 5 spectra collected per well and then averaged for deconvolution. Using normal handshake mode ${ }^{11}$, it took $\sim 20$ min to run a 384 -well plate ( 3 s per sample).

We previously reported using normal handshake mode it took 12 min per 384-well plate (three scans per well) for biochemical assays using IR-MALDESI ${ }^{11}$. We have continued investigating different modes of syncing the mass spectrometer with laser firing for various applications and found that microscan mode can significantly increase the analysis speed. In this mode, one trigger signal was sent to the mass spectrometer to trigger several laser firing and $\mathrm{C}$ trap opening events, the instrument acquires 
multiple spectra and averages them automatically (Figure S5). This increased the data acquisition speed by at least 5 times. Using microscan mode, we were able to scan a full 384-well plate of protein samples in 6.2 minutes with five microscans per well, which converts to $0.97 \mathrm{~s}$ per sample. A test plate was made by alternating cytochrome $\mathrm{C}$ and myoglobin in columns, no cross-contamination between the wells was observed with this mode (Figure S6).

A higher sample speed was achieved using low latency mode coupled with continuous sample stage motion. ${ }^{25}$ In this mode, laser triggering, orbitrap duty cycle and sample stage speed were synced so that one spectrum was acquired per well. A scan of a full 384-well plate, that contained cytochrome $\mathrm{C}$ and water filled in alternating columns, was completed in $17 \mathrm{~s}(22.7 \mathrm{~Hz})$. Even at this increased sampling rate, minimal cross-contamination was observed, and good quality spectra were obtained. Although high speed is generally desirable, averaging spectra is necessary for protein samples at low concentration or in a complex matrix to increase the signal-to-noise ratio. In these instances, microscan or normal handshake modes should be used.

\section{BTK Phosphorylation and Compound Binding Kinetics}

The fast analysis speed of IR-MALDESI can be leveraged to analyze large numbers of samples or to measure fewer samples repetitively. With the tolerance to typical protein buffer systems provided by IR-MALDESI, it is feasible to measure the kinetics of protein - small molecule covalent and noncovalent interactions. A series of experiments were conducted to demonstrate interaction types with various experimental timescales that can be monitored.

One experiment was to determine the autophosphorylation rate of recombinant purified Bruton's tyrosine kinase (BTK) kinase domain. The BTK protein was measured directly in 5 mM HEPES with IRMALDESI, its MS spectrum and deconvoluted spectrum are shown in Figure 3a, the deconvoluted mass was $33505 \mathrm{Da}$. The detection limit of BTK protein was established by a three-fold serial titration experiment, which was $400 \mathrm{nM}$ in $5 \mathrm{mM}$ HEPES buffer (Figure 3b). There is one phosphorylation site in the BTK kinase domain (Y551), ${ }^{26}$ and we observed only one $+80 \mathrm{Da}(33585 \mathrm{Da}$ ) mass shift during the entire time course experiment (Figure 3c). The progress of autophosphorylation was represented by percent mono-phosphorylated (Figure 3d), which was calculated as the ratio of deconvoluted intensity of mono-phosphorylated (reacted) BTK over sum of unreacted and reacted BTK protein. Below 10\% and above $90 \%$ conversion was excluded in the graph because the reacted or unreacted BTK protein fell below the detection limit. All data were collected by repetitively sampling from just three samples. Autophosphorylation was completed in $\sim 3$ hours under these experimental conditions. 
(a)

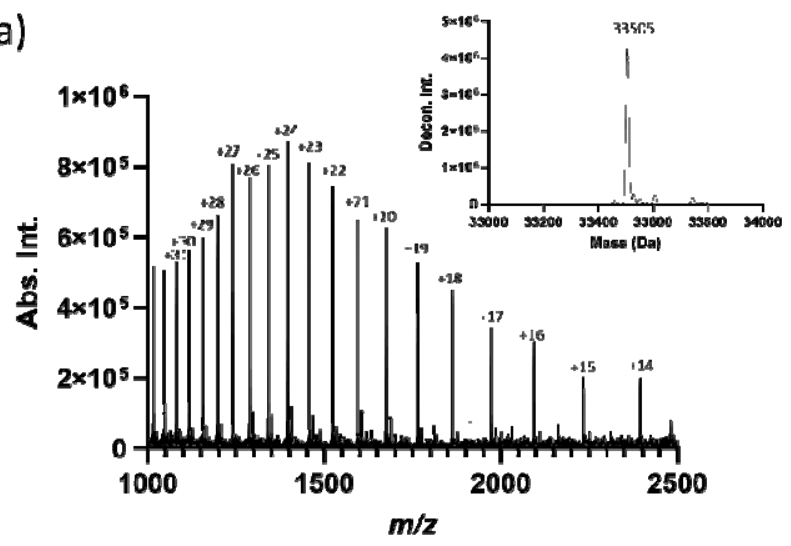

(c)

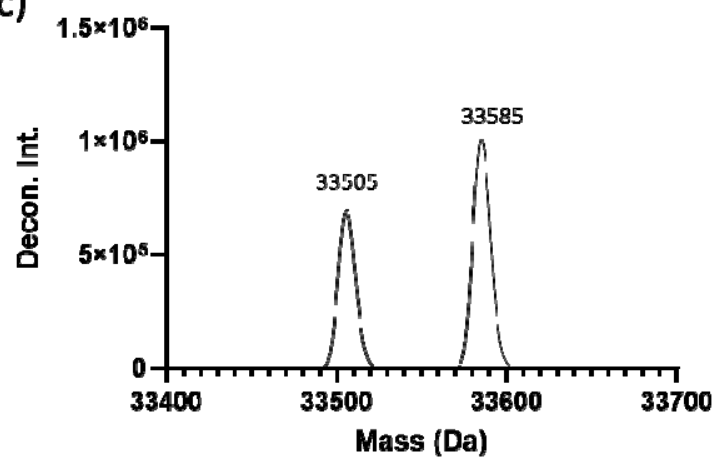

(b)

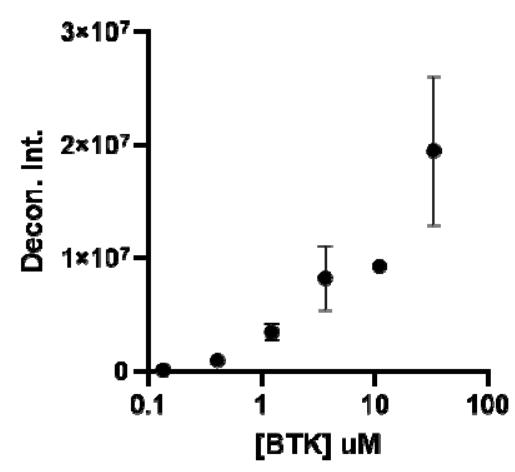

(d)

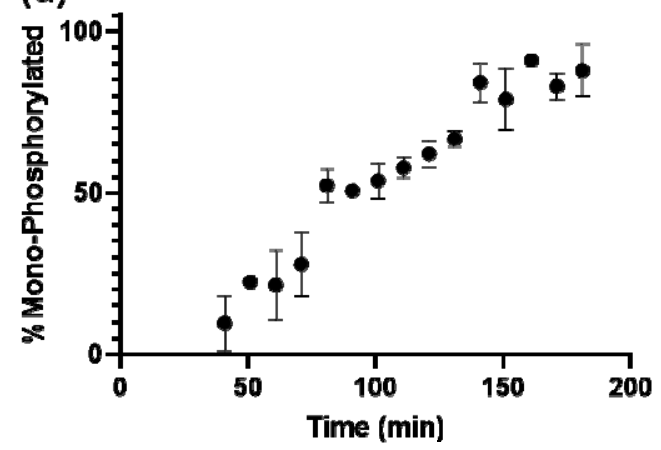

Figure 3. (a) Raw mass spectrum and deconvoluted spectrum (inset) of BTK protein; (b) standard curve of BTK protein in $5 \mathrm{mM}$ HEPES buffer using deconvoluted intensities; (c) deconvoluted spectrum of BTK autophosphorylation in progress; (d) time course of BTK autophosphorylation measured by IR-MALDESI-MS in kinetic mode.

There is renewed interest in covalent inhibitors as potent and effective agents, despite having been deprioritized in drug development in the past due to safety concerns that can be mitigated through careful design ${ }^{27}$. Several covalent inhibitors and non-covalent inhibitors for BTK have been discovered and are approved or in advanced clinical stages ${ }^{28}$. We selected four compounds: acalabrutinib and zanubrutinib (irreversible covalent), rilzabrutinib (reversible covalent), and vecabrutinib (non-covalent) to measure their binding behavior to BTK using IR-MALDESI-MS. Figure S7 shows deconvoluted spectra after incubating BTK with compounds for $10 \mathrm{~min}$ at 1:1 molar ratio. There were no peaks corresponding to compound addition for vecabrutinib (Figure S7a), and the base peaks were still intact protein (33505 $\mathrm{Da})$. This indicates that under our experimental conditions non-covalent complexes were not preserved with IR-MALDESI. For acalabrutinib, zanubrutinib and rilzabrutinib (Figure S7b-d), the compound adduct peaks were the base peaks and their delta masses to intact protein peaks match the compounds' molecular weight (465 Da, $471 \mathrm{Da}$ and $665 \mathrm{Da}$ for acalabrutinib, zanubrutinib and rilzabrutinib, respectively). 
Lowering the $\mathrm{pH}$ of the protein-compound mixture dissociates the reversible covalent complex. By adding an equal volume of $1 \%$ formic acid to the mixture, we observed the compound adduct peak of rilzabrutinib gradually converted to the intact protein peak, hence the percent bound decreased (Figure 4a, calculated as deconvoluted intensity of bound protein over the sum of bound and unbound protein). However, the complex formed between BTK and irreversible covalent compounds remained intact over the similar period (Figure 4b).

By using microscan mode, we demonstrated that it is possible to monitor fast covalent binding events (Figure 4c). Three spectra were collected for each data point, leading to a time resolution of three second. Reaction was initiated manually in a non-reading position, therefore there was $20-30$ s overhead time before the first data point can be taken. This overhead time can be eliminated by dispensing reagent automatically at IR-MALDESI reading position, which is under development. We were not able to capture an association curve for zanubrutinib because the binding ratio reached $100 \%$ within $30 \mathrm{~s}$. However, a complete association curve for acalabrutinib can be obtained and the binding ratio reached $100 \%$ in ca. 5 min.

Due to the irreversible covalent binding of acalabrutinib to BTK, it can serve as a probe to provide insights to kinetics of other noncovalent and reversible covalent binders. In this experiment, vecabrutinib and acalabrutinib were added to BTK solution simultaneously (Figure 4d). The concentration of acalabrutinib was held at a constant $200 \mu \mathrm{M}$ whereas the concentration of vecabrutinib was varied from 0 to $40 \mu \mathrm{M}$. By monitoring the formation of the acalabrutinib-BTK complex, vecabrutinib binding kinetics can be determined by the Motulsky-Mahan model ${ }^{29}$. In the absence of vecabrutinib, acalabrutinib binding to BTK reached maximum in less than 10 min under current assay conditions, whereas the process was significantly slowed down in the presence of vecabrutinib. This allows association/dissociation rates to be estimated for fast binding reactions that could not be monitored without competition. The estimated association $\left(\mathrm{K}_{\mathrm{on}}\right)$ and dissociation rate constant $\left(\mathrm{K}_{\mathrm{off}}\right)$ of vecabrutinib was $\sim 10^{6} \mathrm{M}^{-1} \mathrm{~s}^{-1}$ and $0.03 \mathrm{~s}^{-1}$, respectively. $\mathrm{K}_{\mathrm{D}}$ can be calculated as $\mathrm{K}_{\mathrm{off}} / \mathrm{K}_{\mathrm{on}}$, which was $\sim 30 \mathrm{nM}$. 
(a)

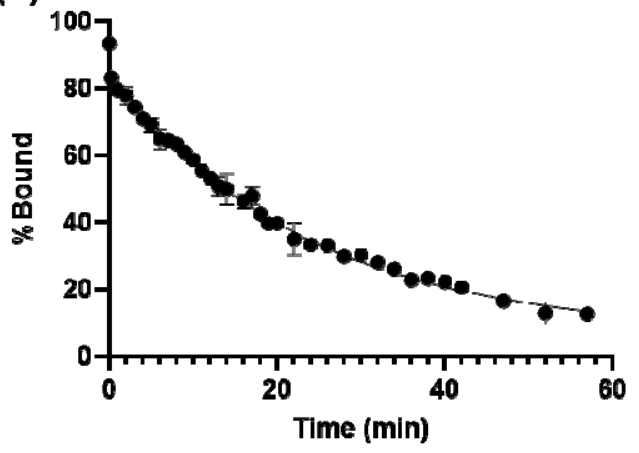

(c)

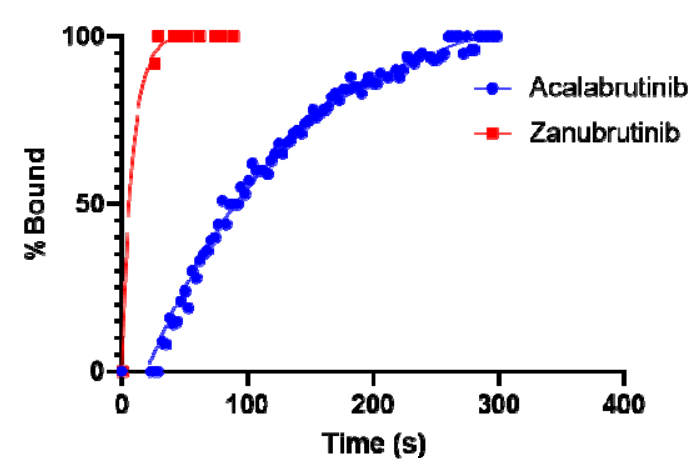

(b)

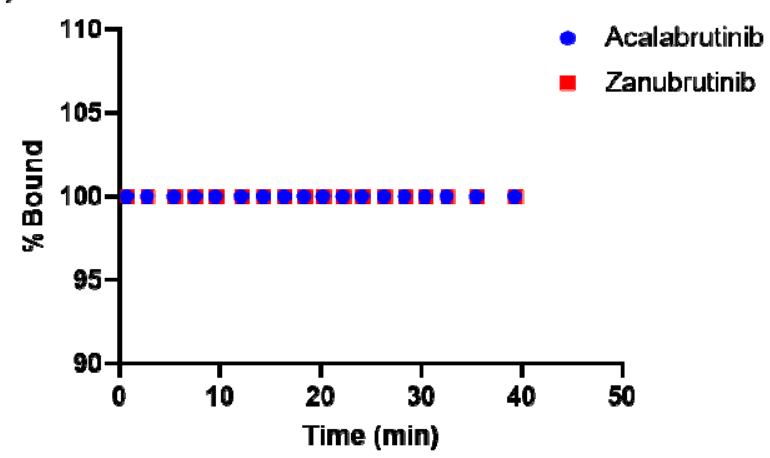

(d)

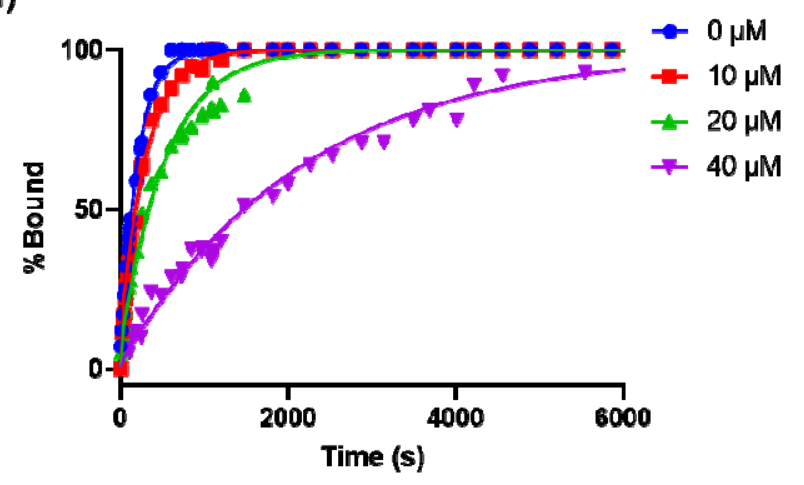

Figure 4. (a) Denaturing of rilzabrutinib and BTK complex via lowering $p H$; (b) lowering $\mathrm{pH}$ did not reverse acalabrutinib/zanubrutinib binding to BTK; (c) monitoring fast covalent binding of acalabrutinib and zanubrutinib to BTK; (d) competitive binding experiment by adding vecabrutinib and acalabrutinib simultaneously to BTK protein, concentrations of vecabrutinib was a variable and reflected as different curves in this figure, $K_{D \text {, vecabrutinib }}=30 \mathrm{nM}$.

\section{Compound Modification to La Protein and Top-down Analysis}

The human La antigen (La protein) is highly reactive to thiol-reactive compounds and can be used to assess compound redox activity to identify false positives from biochemical screens ${ }^{30}$. An assay named ALARM (a La assay to detect reactive molecules) was developed and utilizes either NMR or MS as the detection technique. Here we investigated the potential of developing a high throughput MALDESI ALARM MS assay.

A mass spectrum of unreacted La protein was first collected to establish a reference point and was observed at a deconvoluted mass of $12778 \mathrm{Da}$ (Figure 5a). A series of compounds of different MW, that were reported to be reactive in ALARM $\mathrm{NMR}^{30}$ were incubated with La protein. IR-MALDESI-MS detection was performed after desalting the samples to obtain higher quality spectra. Based on the mass shifts, all tested compounds modified La protein. Hydroquinone formed a direct adduct to La protein (Figure 5b); multiple direct adducts were formed with 2-chloronaphthalene-1,4-dione (Figure 5c) and ethacrynic acid (Figure 5d); cefaclor formed a direct adduct while oxidation (2O) peak was also detected (Figure 5e); omeprazole formed an adduct with La protein through formation of disulfide bond (Figure $5 f)$.

From intact protein analysis we obtained information on the modification type and extent from incubating La protein with each test compound. To gain additional insights on modification location, top- 
down protein sequencing using tandem mass spectrometry (MS/MS) can be performed on the sample. ${ }^{31}$ As a proof-of-concept, we incubated La protein with hydroquinone and performed top-down analysis using high energy collision induced dissociation (HCD). MS/MS spectra were collected at ten NCEs (normalized collision energies) from 20 to 29 on peak $\mathrm{m} / \mathrm{z} 990(\mathrm{z}=13)$. Data analysis indicated that the hydroquinone modification site was located at C23 even though sequence coverage was incomplete (Figure S8). When the modification was placed in silico at C23 instead of C10, coverage increased from $38 \%$ to $41 \%$, with four additional assignable fragment ions residing between the two cysteines indicating $\mathrm{C} 23$ was the modification site.

(a)

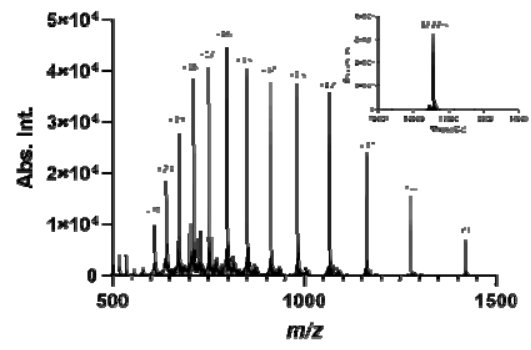

(d)

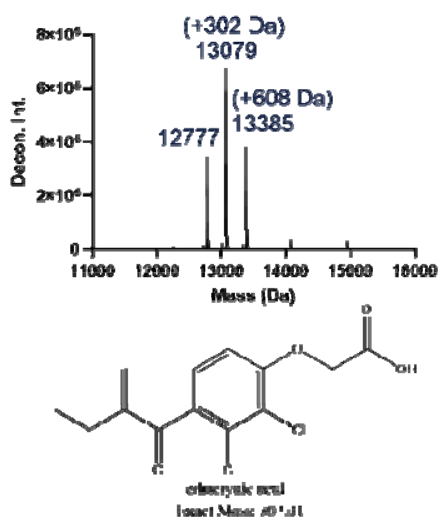

(b)

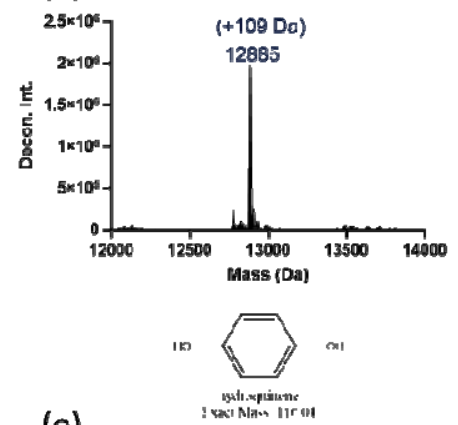

(e)

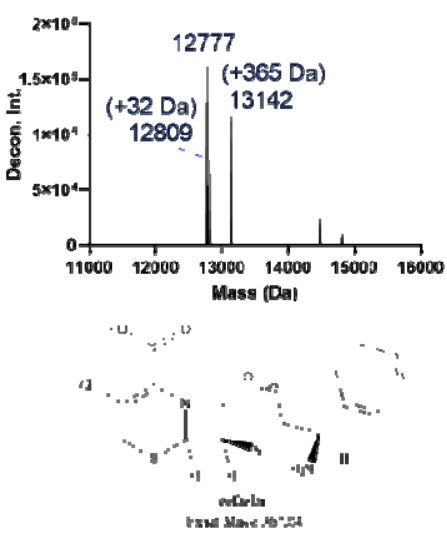

(c)

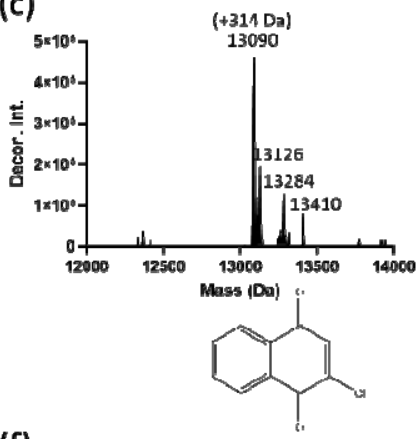

(f)
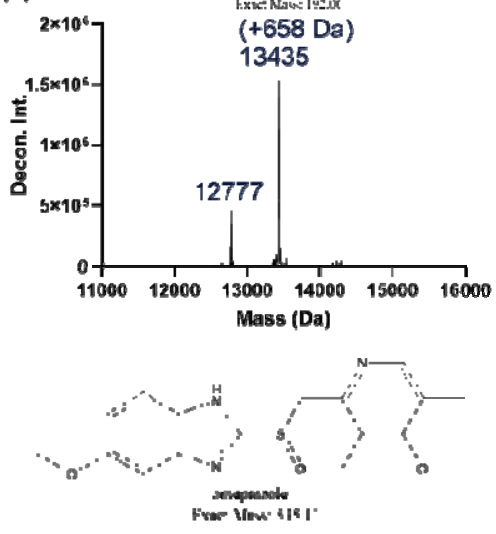

Figure 5. ALARM MS using IR-MALDESI-MS. (a) full and deconvoluted spectrum of unreacted La protein; deconvoluted spectra and chemical structures of (b) hydroquinone; (c) 2-chloronaphthalene-1,4-dione; (d) ethacrynic acid; (e) cefaclor and (f) omeprazole.

\section{High Throughput Sample Cleanup Coupled with IR-MALDESI-MS}

As we have established earlier in this paper, many salts/buffers have concentration dependent ion suppression effects even at relatively high amounts of salt. For some applications, high salt/buffer concentrations and relatively low protein concentrations requires sample cleanup. Therefore, a quick, multiplexed sample cleanup step could greatly expand the applicability of IR-MALDESI. For example, since the incubation media for ALARM MS was PBS, desalting had to be performed to obtain high quality spectra to ease data interpretation. There are many strategies to perform high throughput desalting of individual protein samples, most of which are commercially available. Here we demonstrate the use of two plate-based strategies with IR-MALDESI-MS: slit plate and magnetic beads. 
Slit plate is a commercially available desalting plate based on similar principle as TopTip. Micrometer sized slits cut at the bottom of wells and can be filled with different stationary phase chromatographic materials. We used a C4 slit plate to clean up $0.3 \mathrm{mg} / \mathrm{mL}$ La proteins spiked in PBS. Different equilibration and wash cycles were compared. Samples were eluted to a PCR plate and IRMALDESI was performed directly from the PCR plate. Significant difference between the mass spectra could be observed before and after cleanup (Figure $6 \mathrm{a}$ and $6 \mathrm{~b}$ ). The signal to noise ratios (SNR) of +16 peak $(\mathrm{m} / \mathrm{z}$ 797) were used to assess signal recovery after clean-up. Three equilibration and wash cycles were recommended by the vendor but even with only one equilibration and one wash cycle, significant signal recovery was achieved (Figure S9). Considering that each cycle would require separate centrifugation, reducing the number of cycles would result in higher throughput while maintaining sufficient performance. Depending on the number of equilibration/wash cycles, it would take ca. 10-30 min to process one 384-well plate.

Magnetic beads are another fast and flexible method to perform sample cleanup. They can be coated with different materials such as reversed phase chromatography materials, Ni-NTA, and protein A to either desalt or isolate and concentrate a protein of interest. After protein binding, the beads can be collected with magnets and washes can be performed to remove unwanted species. The sample cleanup can be automated with many types of liquid handling equipment. Here we performed a proof-of-concept experiment using C-18 coated magnetic beads (BcMag C-18) on a Blue Washer (BlueCatBio). $1 \mathrm{mg} / \mathrm{mL}$ rHSA was spiked into PBS as the simulated protein sample for cleanup. After magnetic beads cleanup with Blue Washer, better SNR and protein envelope could be observed when comparing to direct sampling from PBS (Figure $6 \mathrm{c}$ and $6 \mathrm{~d}$ ). We were able to deconvolute the mass spectrum with high confidence after cleanup. Magnetic beads cleanup with Blue Washer does not require any separate centrifugation or dispensing steps as magnetic carriers were available to collect beads at the bottom of the plates, and the device could be programmed to evacuate/dispense liquid. A full protocol executed on the Blue Washer (excluding sample dispensing) required $<10 \mathrm{~min}$. Considering the flexibility of mechanisms magnetic beads provide, it is a highly versatile platform to perform sample cleanup for IR-MALDESI or incorporate into more complex sample work-up procedures. An example of different sample work-up was performed using protein A magnetic beads to capture NISTmAb spiked into PBS (Figure S10). 
(a)

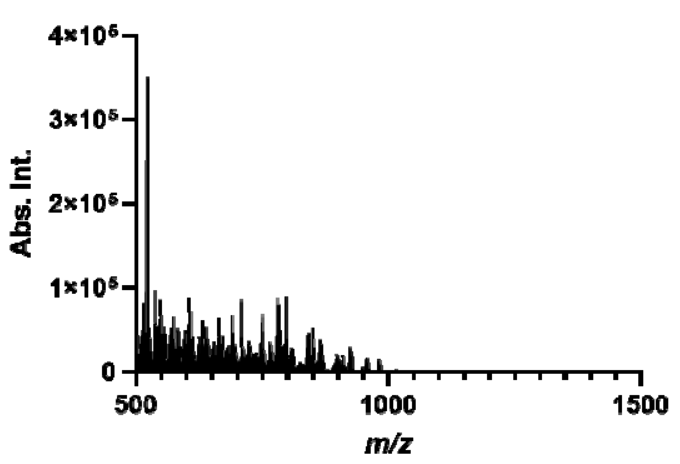

(c)

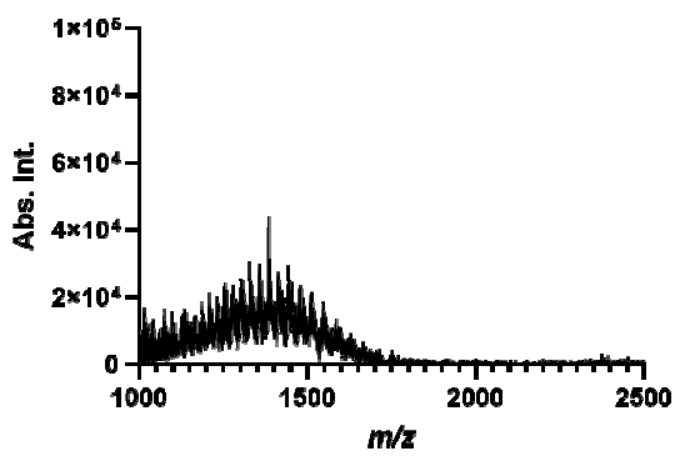

(b)

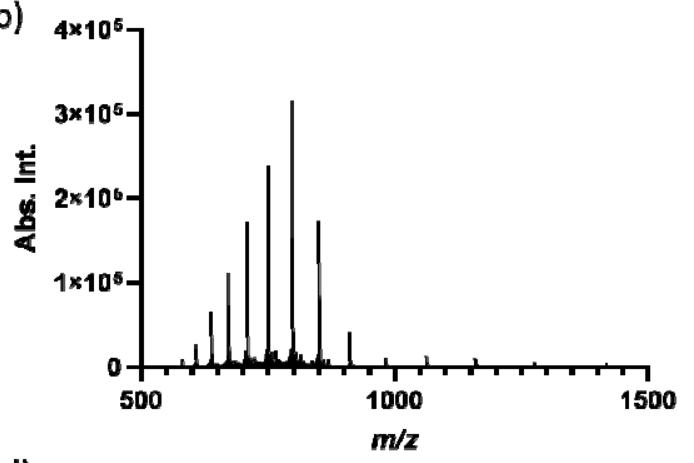

(d)

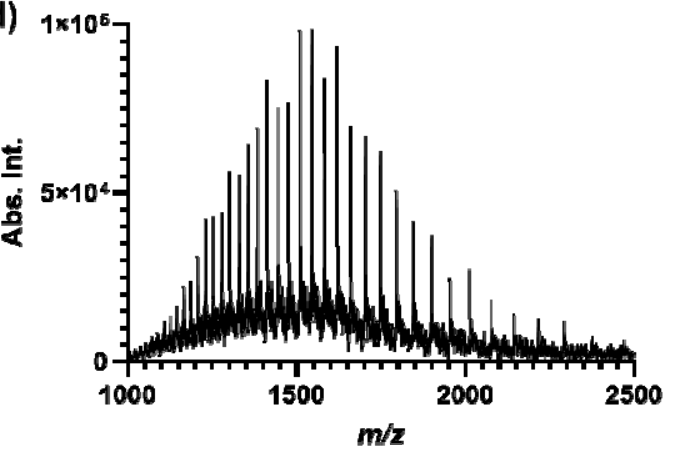

Figure 6. High throughput desalting coupled with IR-MALDESI-MS. Before (a) and after $(b)$ slit plate desalting of La protein in PBS; before (c) and after (d) C-18 magnetic beads desalting of rHSA in PBS.

\section{Conclusions:}

In this work, we demonstrated the use of a high throughput IR-MALDESI-MS system to perform protein analysis. Proteins up to $150 \mathrm{kDa}$ could be detected and we established matrix effects caused by several common buffer components. By using microscan mode, the run time of a 384-well plate was 6.2 min, or less than one second per sample. Even higher speed at 22 samples per second was demonstrated by low latency mode using continuous stage motion. Besides analyzing a large quantity of samples, the high analysis speed was utilized to study kinetics, as demonstrated by BTK autophosphorylation and compound binding experiments. We have also run ALARM assay with IR-MALDESI-MS and top-down protein sequencing was performed on the same sample to identify the cysteine modification site. High throughput desalting strategies, based on multi-well microtiter plates, were applied to clean up samples in a 384 well plate without significantly sacrificing throughput using slit plate and magnetic beads. The most significant bottleneck we are experiencing is data analysis, since it was done on a sample-per-sample basis rather than on a plate-per-plate basis. Besides applications discussed in this work, we have also explored additional applications to support drug discovery efforts, such as covalent fragment screening and compound induced target protein modifications (data not shown). Native MS with IR-MALDESI is another important future direction and was recently demonstrated by analyzing NISTmAb on an Orbitrap Exploris $^{\mathrm{TM}} 240$ mass spectrometer ${ }^{32}$. Native MS performed on our IR-MALDESI-MS system was used to examine a protein-ligand complex, acetazolamide non-covalently bound to carbonic anhydrase (Figure S11). We believe IR-MALDESI-MS is a true versatile high throughput platform that can be used widely for protein analysis applications. 


\section{Methods:}

\section{Materials:}

All chemicals except the following were purchased from Sigma-Aldrich: hydroquinone was purchased from ACROS Organics, 2-chloro-1,4-naphthoquinone was purchased from TCI America, ultrapure ATP was purchased from Promega, 1 M HEPES pH 8 was purchased from Teknova. The following protein standards were purchased from Sigma-Aldrich: cytochrome C (C2037, from bovine heart), carbonic anhydrase (C3934, from bovine erythrocytes), rHSA (A6608) and myoglobin (M0630, from equine skeletal muscle).

Drug compounds were obtained from AbbVie compound repository. C-18 BcMag magnetic beads were purchased from Bioclone, C-4 TopTip and C-4 slit plate were purchased from Glysci. Pierce Protein A magnetic beads, Zeba Spin Column (7K MWCO) and protein concentrator (10K MWCO) were purchased from ThermoFisher. BTK kinase domain (387-659) and HCV-La protein were obtained from AbbVie. BTK protein were concentrated to ca. $100 \mathrm{uM}$ and buffer exchanged to $5 \mathrm{mM}$ HEPES pH 7.5 using protein concentrator. Intact protein analysis of La protein was performed using ${ }^{13} \mathrm{C}$ labeled protein that was also routinely used for ALARM NMR, whereas top-down analysis was performed using unlabeled La protein, their sequences were identical. All protein analysis were performed on 384 well small volume HiBase microplates (Greiner Bio-One, 784075) except for desalting experiments. Protein desalting on Bluewasher and elution from slit plate were performed using 384 well Hard-Shell PCR plates (Bio-Rad, HSP-3805), the PCR plates were used for IR-MALDESI-MS readout directly without transfer.

\section{Instrumentation:}

The high throughput IR-MALDESI-MS system was described previously ${ }^{11}$. Briefly, a $2970 \mathrm{~nm}$ laser was used to energize samples and an electrospray emitter aligned to the MS inlet was used to ionize the neutral laser plume. The IR-MALDESI source was coupled to Q Exactive HF-X (Thermo Fisher) mass spectrometer. An extended capillary with custom cartridge heater and motorized stage were used to accommodate analysis of standard microtiter plates.

\section{MS Conditions:}

A resolving power of $7,500(\mathrm{FWHM}$ at $\mathrm{m} / \mathrm{z}=200)$ was used for intact protein analysis except for topdown experiment, where a resolving power of 240, 000 (FWHM at $\mathrm{m} / \mathrm{z}=200$ ) was used. A spray voltage of $4 \mathrm{kV}$ was supplied to the ESI solvent, which was $80 / 20$ methanol/water v/v with $0.1 \%$ formic acid. ESI flow rate was $2 \mu \mathrm{L} / \mathrm{min}$. Capillary temperature was set at $400{ }^{\circ} \mathrm{C}$ and temperature of the extended capillary was set at $120{ }^{\circ} \mathrm{C}$.

\section{BTK Experiments:}

BTK phosphorylation experiment was performed in a buffer made of $5 \mathrm{mM}$ HEPES pH 7.5, $0.5 \mathrm{mM}$ $\mathrm{MgCl}_{2}, 75 \mathrm{mM}$ ammonium acetate and $0.5 \mathrm{mM}$ ATP. $50 \mu \mathrm{M}$ of BTK was used.

BTK binding experiment was performed in 5mM HEPES pH 7.5. $10 \mathrm{mM}$ compounds stock solutions were diluted to desired concentrations with the buffer. $10 \mu \mathrm{L}$ of $35 \mu \mathrm{M}$ BTK was first added to wells then equal volume of $2 \mathrm{X}$ compound solution was added to initiate reaction. For denaturing BTK equal volume of $1 \%$ formic acid was added to the BTK-compound solution.

IR-MALDESI readout was performed from the buffer directly and in real time. 


\begin{abstract}
ALARM MS:
La protein was first buffer exchanged to PBS to remove residual dithiothreitol in the storage buffer. 0.16 $\mathrm{mg} / \mathrm{mL}$ La protein was incubated with $500 \mu \mathrm{M}$ compounds for $1 \mathrm{~h}$ then desalted with $\mathrm{C} 4$ TopTip before IR-MALDESI-MS analyses.
\end{abstract}

\title{
Protein Desalting with BcMag Magnetic Beads:
}

Magnetic beads were suspended into $50 / 50$ methanol/water $\mathrm{v} / \mathrm{v}$ at $50 \mathrm{mg} / \mathrm{mL}$ and stored at $4{ }^{\circ} \mathrm{C}$. 10 $\mu \mathrm{L} /$ well of the stock mixture was added to plates and storage solution was evacuated on the Blue washer on a magnetic carrier. $30 \mu \mathrm{L} /$ well equilibration buffer $(0.5 \%$ trifluoroacetic acid, $95 / 5$ water/acetonitrile $\mathrm{v} / \mathrm{v}$ ) was added and removed to equilibrate magbeads. $30 \mu \mathrm{L}$ protein sample was mixed with $10 \mu \mathrm{L}$ sampling binding buffer ( $2 \%$ trifluoroacetic acid, 95/5 water/acetonitrile $\mathrm{v} / \mathrm{v}$ ) and incubated for $1 \mathrm{~min}$ before adding to working plate. Washing was performed three times with equilibration buffer then $30 \mu \mathrm{L}$ elution buffer (50/50 acetonitrile/water) was added to release protein. Desalted samples were eluted to PCR plate for IR-MALDESI-MS analyses.

\section{Protein Desalting with Slit Plate:}

Slit plate works like TopTip, except that it is plate-based and available in 384-well format. The plate was first equilibrated with elution buffer $(0.1 \%$ formic acid, $50 / 50$ acetonitrile/water) for three times followed by three equilibration cycles with binding buffer $(0.1 \%$ formic acid). Protein samples were added after equilibration and spun down, followed by three washes with binding buffer and elute with elution buffer. Each wash/equilibration cycle involved centrifuge at $1000 \mathrm{~g}$ for $1 \mathrm{~min}$ except for the last sample elution step, where the plate was centrifuged at $1000 \mathrm{~g}$ for $2 \mathrm{~min}$.

\section{Data Analysis:}

Protein MS deconvolution was performed using ReSpect algorithm in Biopharma Finder (Thermo Fisher). Top-down analysis was also conducted with Biopharma Finder. Model fitting and other plots were made with GraphPad Prism 9.

\section{Acknowledgements:}

The authors acknowledge Laura J. Miesbauer (AbbVie), Robert W. Johnson (AbbVie), Kevan Krizner (NCSU), Ken Garrard (NCSU), M. Caleb Bagley (NCSU), David C. Muddiman (NCSU) for their critical insights. Funding was provided to David C. Muddiman (NCSU) by AbbVie. There is no funding to disclose for Kevan Krizner (NCSU), Ken Garrard (NCSU) and M. Caleb Bagley (NCSU). The work was enabled by the AbbVie Postdoc Program. All authors are employees of AbbVie. The design, study conduct, and financial support for this research were provided by AbbVie. AbbVie participated in the interpretation of data, review, and approval of the publication.

\section{References}

1. Fenn, J. B.; Mann, M.; Meng, C. K.; Wong, S. F.; Whitehouse, C. M., Electrospray ionization for mass spectrometry of large biomolecules. Science 1989, 246 (4926), 64-71. 
2. $\quad$ Karas, M.; Hillenkamp, F., Laser desorption ionization of proteins with molecular masses exceeding 10,000 daltons. Anal Chem 1988, 60 (20), 2299-301.

3. Domon, B.; Aebersold, R., Mass spectrometry and protein analysis. Science 2006, 312 (5771), 212-7.

4. White, W. L.; Wagner, C. D.; Hall, J. T.; Chaney, E. E.; George, B.; Hofmann, K.; Miller, L. A.; Williams, J. D., Protein open-access liquid chromatography/mass spectrometry. Rapid Commun Mass Spectrom 2005, 19 (2), 241-9.

5. Campuzano, I. D.; San Miguel, T.; Rowe, T.; Onea, D.; Cee, V. J.; Arvedson, T.; McCarter, J. D., High-Throughput Mass Spectrometric Analysis of Covalent Protein-Inhibitor Adducts for the Discovery of Irreversible Inhibitors: A Complete Workflow. J Biomol Screen 2016, 21 (2), 136-44.

6. $\quad$ Sawyer, W. S.; Srikumar, N.; Carver, J.; Chu, P. Y.; Shen, A.; Xu, A.; Williams, A. J.; Spiess, C.; Wu, C.; Liu, Y.; Tran, J. C., High-throughput antibody screening from complex matrices using intact protein electrospray mass spectrometry. Proc Natl Acad Sci U S A 2020, 117 (18), 98519856.

7. Park, H. M.; Winton, V. J.; Drader, J. J.; Manalili Wheeler, S.; Lazar, G. A.; Kelleher, N. L.; Liu, Y.; Tran, J. C.; Compton, P. D., Novel Interface for High-Throughput Analysis of Biotherapeutics by Electrospray Mass Spectrometry. Anal Chem 2020, 92 (2), 2186-2193.

8. Bachman, M.; Sinclair, I.; Ivanov, D.; Wingfield, J., Information-rich high-throughput cellular assays using acoustic mist ionisation mass spectrometry. Analyst 2021, 146 (1), 315-321.

9. Zhang, H.; Liu, C.; Hua, W.; Ghislain, L. P.; Liu, J.; Aschenbrenner, L.; Noell, S.; Dirico, K. J.; Lanyon, L. F.; Steppan, C. M.; West, M.; Arnold, D. W.; Covey, T. R.; Datwani, S. S.; Troutman, M. D., Acoustic Ejection Mass Spectrometry for High-Throughput Analysis. Anal Chem 2021, 93 (31), 10850-10861.

10. Morato, N. M.; Holden, D. T.; Cooks, R. G., High-Throughput Label-Free Enzymatic Assays Using Desorption Electrospray-Ionization Mass Spectrometry. Angew Chem Int Ed Engl 2020, 59 (46), 20459-20464.

11. Pu, F.; Radosevich, A. J.; Sawicki, J. W.; Chang-Yen, D.; Talaty, N. N.; Gopalakrishnan, S. M.; Williams, J. D.; Elsen, N. L., High-Throughput Label-Free Biochemical Assays Using Infrared Matrix-Assisted Desorption Electrospray Ionization Mass Spectrometry. Anal Chem 2021, 93 (17), 6792 6800.

12. Miao, Z.; Chen, H., Direct analysis of liquid samples by desorption electrospray ionization-mass spectrometry (DESI-MS). J Am Soc Mass Spectrom 2009, 20 (1), 10-9.

13. Shin, Y. S.; Drolet, B.; Mayer, R.; Dolence, K.; Basile, F., Desorption electrospray ionizationmass spectrometry of proteins. Anal Chem 2007, 79 (9), 3514-8.

14. Ferguson, C. N.; Benchaar, S. A.; Miao, Z.; Loo, J. A.; Chen, H., Direct ionization of large proteins and protein complexes by desorption electrospray ionization-mass spectrometry. Anal Chem 2011, 83 (17), 6468-73.

15. Garza, K. Y.; Feider, C. L.; Klein, D. R.; Rosenberg, J. A.; Brodbelt, J. S.; Eberlin, L. S., Desorption Electrospray Ionization Mass Spectrometry Imaging of Proteins Directly from Biological Tissue Sections. Anal Chem 2018, 90 (13), 7785-7789.

16. Ambrose, S.; Housden, N. G.; Gupta, K.; Fan, J.; White, P.; Yen, H. Y.; Marcoux, J.; Kleanthous, C.; Hopper, J. T. S.; Robinson, C. V., Native Desorption Electrospray Ionization Liberates Soluble and Membrane Protein Complexes from Surfaces. Angew Chem Int Ed Engl 2017, 56 (46), 14463-14468.

17. Yan, B.; Bunch, J., Probing Folded Proteins and Intact Protein Complexes by Desorption Electrospray Ionization Mass Spectrometry. J Am Soc Mass Spectrom 2021, 32 (3), 690-699.

18. Hsu, C. C.; Chou, P. T.; Zare, R. N., Imaging of Proteins in Tissue Samples Using Nanospray Desorption Electrospray Ionization Mass Spectrometry. Anal Chem 2015, 87 (22), 11171-5.

19. Chen, C. L.; Kuo, T. H.; Chung, H. H.; Huang, P.; Lin, L. E.; Hsu, C. C., Remodeling nanoDESI Platform with Ion Mobility Spectrometry to Expand Protein Coverage in Cancerous Tissue. $J$ Am Soc Mass Spectrom 2021, 32 (3), 653-660. 
20. Hale, O. J.; Cooper, H. J., Native Mass Spectrometry Imaging of Proteins and Protein Complexes by Nano-DESI. Anal Chem 2021, 93 (10), 4619-4627.

21. Sampson, J. S.; Hawkridge, A. M.; Muddiman, D. C., Generation and detection of multiplycharged peptides and proteins by matrix-assisted laser desorption electrospray ionization (MALDESI) Fourier transform ion cyclotron resonance mass spectrometry. J Am Soc Mass Spectrom 2006, 17 (12), 1712-6.

22. Sampson, J. S.; Murray, K. K.; Muddiman, D. C., Intact and top-down characterization of biomolecules and direct analysis using infrared matrix-assisted laser desorption electrospray ionization coupled to FT-ICR mass spectrometry. J Am Soc Mass Spectrom 2009, 20 (4), 667-73.

23. Nemes, P.; Vertes, A., Laser ablation electrospray ionization for atmospheric pressure, in vivo, and imaging mass spectrometry. Anal Chem 2007, 79 (21), 8098-106.

24. Ekelof, M.; McMurtrie, E. K.; Nazari, M.; Johanningsmeier, S. D.; Muddiman, D. C., Direct Analysis of Triterpenes from High-Salt Fermented Cucumbers Using Infrared Matrix-Assisted Laser Desorption Electrospray Ionization (IR-MALDESI). J Am Soc Mass Spectrom 2017, 28 (2), 370-375. 25. Radosevich, A. J.; Pu, F.; Chang-Yen, D.; Sawicki, J. W.; Talaty, N. N.; Elsen, N. L.; Williams, J. D.; Pan, J. Y., Ultrahigh-Throughput Direct Sampling MS: Sampling at $22 \mathrm{~Hz}$ by Infrared Matrix-Assisted Desorption Electrospray Ionization Mass Spectrometry BioRxiv 2021.

26. Rawlings, D. J.; Scharenberg, A. M.; Park, H.; Wahl, M. I.; Lin, S.; Kato, R. M.; Fluckiger, A. C.; Witte, O. N.; Kinet, J. P., Activation of BTK by a phosphorylation mechanism initiated by SRC family kinases. Science 1996, 271 (5250), 822-5.

27. Singh, J.; Petter, R. C.; Baillie, T. A.; Whitty, A., The resurgence of covalent drugs. Nat Rev Drug Discov 2011, 10 (4), 307-17.

28. Gu, D.; Tang, H.; Wu, J.; Li, J.; Miao, Y., Targeting Bruton tyrosine kinase using non-covalent inhibitors in B cell malignancies. J Hematol Oncol 2021, 14 (1), 40.

29. Motulsky, H. J.; Mahan, L. J. M. p., The kinetics of competitive radioligand binding predicted by the law of mass action. 1984, 25 (1), 1-9.

30. Huth, J. R.; Mendoza, R.; Olejniczak, E. T.; Johnson, R. W.; Cothron, D. A.; Liu, Y.; Lerner, C. G.; Chen, J.; Hajduk, P. J., ALARM NMR: a rapid and robust experimental method to detect reactive false positives in biochemical screens. J Am Chem Soc 2005, 127 (1), 217-24.

31. Siuti, N.; Kelleher, N. L., Decoding protein modifications using top-down mass spectrometry. Nat Methods 2007, 4 (10), 817-21.

32. Knizner, K. T.; Bagley, M.C.; Garrard, K.P.; Pu, F.; Elsen, N.L.; Williams, J.D.; Muddiman, D.C., Optimized C-trap Timing of an Orbitrap Mass Spectrometer for General High Throughput Screening Analyses by IR-MALDESI Submitted 2021. 